\title{
Charta zur ärztlichen Berufsethik
}

\author{
Einleitung
}

Immer wieder wird gefordert, dass sich die Ärzte mit grundlegenden Fragen ihrer Berufsausübung auseinandersetzen und die fundamentalen Verantwortlichkeiten und ethischen Prinzipien zu definieren versuchen.

Unter dem Titel «Medical Professionalism in the New Millennium - a Physician Charter» haben drei internistische Organisationen einen solchen fächerübergreifenden Text erarbeitet. Es handelt sich um die European Federation of Internal Medicine, das American College of Physicians und den American Board of Internal Medicine. Die Schweizerische Gesellschaft für Innere Medizin (SGIM) ist Mitglied der Europäischen Internistenföderation und hat bei der Formulierung mitwirken können.

Die Charta hat nach der Erstpublikation (2002) in den Annals of Internal Medicine [1] und im Lancet [2] weltweit grosse Beachtung gefunden, wurde bereits in vielen Zeitschriften nachgedruckt, in mehrere Sprachen übersetzt und vor allem in England, Skandinavien und
Amerika auch im Rahmen von Veranstaltungen für Ärzte, Studenten und Laien diskutiert.

So hat zum Beispiel das italienische Gesundheitsministerium das Dokument an alle Praktiker und Fakultätsmitglieder des Landes verschickt.

Die Charta ist der internationale Versuch, historische Dokumente wie den «Eid des Hippokrates» durch einen Text zu ergänzen, der den Herausforderungen der heutigen Zeit an Ärztinnen und Ärzte gerecht wird.

Dr. Werner Bauer Präsident SGIM

1 ABIM Foundation. American Board of Internal Medicine; ACP-ASIM Foundation. American College of Physicians-American Society of Internal Medicine; European Federation of Internal Medicine. Medical professionalism in the new millennium: a physician charter. Ann Intern Med. 2002 Feb 5;136(3):243-6.

2 Medical professionalism in the new millennium: a physicians' charter. Medical Professionalism Project. Lancet 2002;359:520-522

Charta zur ärztlichen Berufsethik: Seiten 2347-49. 